\title{
Agreement of Corneal Endothelial Cell Analysis Between Konan-Noncon Robo SP-6000 and Tomey EM-3000 Specular Microscopes in Healthy Subjects
}

\author{
Jbara, Doha
}

2021-04

Jbara , D , Achiron , A , Antman , G, Buhbut , O , Hecht , I , Tuuminen , R , Bahar , I \& Elbaz , U 2021, ' Agreement of Corneal Endothelial Cell Analysis Between Konan-Noncon Robo SP-6000 and Tomey EM-3000 Specular Microscopes in Healthy Subjects ' , Eye and Contact Lens, vol. 47 , no. 4 , pp. 191-195 . https://doi.org/10.1097/ICL.0000000000000712

http://hdl.handle.net/10138/332975

https://doi.org/10.1097/ICL.0000000000000712

cc_by_nc_nd

publishedVersion

Downloaded from Helda, University of Helsinki institutional repository.

This is an electronic reprint of the original article.

This reprint may differ from the original in pagination and typographic detail.

Please cite the original version. 


\title{
Agreement of Corneal Endothelial Cell Analysis Between Konan-Noncon Robo SP-6000 and Tomey EM-3000 Specular Microscopes in Healthy Subjects
}

\author{
Doha Jbara, M.D., Asaf Achiron, M.D., Gal Antman, M.D., Ortal Buhbut, M.D., Idan Hecht, M.D., \\ Raimo Tuuminen, M.D., Ph.D., Irit Bahar, M.D., M.H.A., and Uri Elbaz, M.D.
}

\begin{abstract}
Purpose: To compare corneal endothelium parameters taken by two common noncontact specular microscopes in healthy subjects.

Methods: Healthy participants visiting the outpatient eye clinic at the Rabin Medical Center, Petah Tikva, Israel, were recruited prospectively. All participants underwent three consecutive corneal endothelial cell photographs with both the Konan-Noncon Robo SP-6000 and the Tomey EM-3000 specular microscopes. Endothelial cell density (ECD) was evaluated using the manual center technique in both machines. Bland-Altman graphs were used to assess the agreement between the devices, and intraclass correlation coefficient (ICC) served to assess intraobserver variability for each device. Results: Recruited were 49 healthy subjects with a mean age of $48.9 \pm 15.6$ years, 49 right eyes were included. The mean ECD was comparable between the Tomey EM-3000 and the Konan-Noncon Robo SP-6000 $\left(2,713.2 \pm 242.4\right.$ vs. $2,700.8 \pm 300.5$ cells $/ \mathrm{mm}^{2}$, respectively, $\left.P=0.47\right)$ with a mean difference of 12.4 cells $/ \mathrm{mm}^{2}(0.67 \%)$, a mean ECD absolute difference of 93.3 cells $/ \mathrm{mm}^{2}$, and low $95 \%$ limits of agreement of -222.0 to +246.9 cells $/ \mathrm{mm}^{2}$. A folded empirical distribution function curve showed that all differences fell within 525.4 cells $/ \mathrm{mm}^{2}$, centered around a median of 13.3 cells $/ \mathrm{mm}^{2}$. Intraclass correlation coefficient was high for both the Konan-Noncon Robo SP-6000 (0.93, 95\% confidence interval [CI]: $0.89-0.95)$ and the Tomey EM-3000 (0.88, 95\% CI: 0.82-0.93).

Conclusions: The difference in endothelial cell measurements between the Konan SP-6000 and the Tomey EM-3000 specular microscopes through the center and the L-count analyzing techniques, respectively, is clinically small and not statistically significant. Nevertheless, caution should be taken when used interchangeably because ECD difference between the two machines can be as high as 525.4 cells $/ \mathrm{mm}^{2}$.
\end{abstract}

From the Department of Ophthalmology (D.J., G.A., I.B., U.E.), Rabin Medical Center, Petah-Tikva, Israel; Department of Ophthalmology, (D.J., A.A., G.A., I.H., I.B., U.E.), Sackler Faculty of Medicine, Tel Aviv University, Tel Aviv, Israel; Department of Ophthalmology (A.A.), Edith Wolfson Medical Center, Holon, Israel; Department of Ophthalmology (O.B.), Soroka University Medical Center, The Faculty of Health Sciences, Ben-Gurion University of the Negev, Beer-Sheva, Israel; Department of Ophthalmology (I.H.), Shamir Medical Center, Be'er Ya'akov, Israel; Department of Ophthalmology (R.T.), Helsinki Retina Research Group, University of Helsinki, Helsinki, Finland; and Eye Centre (R.T.), Kymenlaakso Central Hospital, Kotka, Finland.

The authors have no funding or conflicts of interest to disclose

Supplemental digital content is available for this article. Direct URL citations appear in the printed text and are provided in the HTML and PDF versions of this article on the journal's Web site (www. eyeandcontactlensjournal.com).

Address correspondence to Uri Elbaz, M.D., Department of Ophthalmology, Rabin Medical Center, Petah-Tikva, Israel 39, Ze'ev Jabotinsky Street, Petah-Tikva, Israel 4941492; e-mail: urielbaz@gmail.com

Accepted March 22, 2020.

DOI: 10.1097/ICL.0000000000000712
Key Words: Specular microscopy—Endothelial cell count-Tomey EM3000-Konan-Noncon Robo SP-6000.

(Eye \& Contact Lens 2021;47: 191-195)

$\mathrm{H}$ uman corneal endothelial cells form a monolayer of uniformly sized and semipermeable hexagonal-shaped cells, sitting on the inner surface of the human cornea. ${ }^{1-3}$ They are able to maintain a transparent and clear cornea due to their ability to function as a passive barrier and also are able to actively pump the aqueous humor. ${ }^{4,5}$ With aging, there is a gradual decline in the cell density at an estimated annual rate of $0.6 \%{ }^{6,7}$ because human endothelial cells lack the capability to replicate and regenerate. The rate can accelerate in the presence of ocular morbidities, such as corneal endothelial dystrophies, corneal trauma, and after routine surgical procedures, including cataract extraction or other intraocular surgeries. ${ }^{8}$ Therefore, evaluating endothelial cell density (ECD) accurately is of a high importance in routine practice and for preoperative and postoperative assessments.

The Konan-Noncon Robo SP-6000 (Konan Medical, Hyogo, Japan) and Tomey EM-3000 (Tomey, Nagoya, Japan) are both widely used for assessing ECD and health of the endothelium in clinical practice; however, only the Konan-Noncon Robo SP-6000 specular microscope (SM) is FDA-approved. ${ }^{9}$

There is paucity of data comparing these two devices. To the best of our knowledge, there are only two studies (Luft et al. ${ }^{8}$ and Price et al. ${ }^{10}$ ) comparing the efficacy of these two devices in assessing endothelial cell parameters, conducted in mixed cohorts of healthy and abnormal corneas. However, although both the manual and automated techniques were used with the Konan microscope, only the automated technique was used with the Tomey EM-3000 microscope because image analysis could not be performed manually in those studies. Because manual technique for ECD estimation was shown to be more accurate, ${ }^{10}$ we aim in this study to compare the two SMs using manual counting methods only, for images taken with both devices in healthy participants solely.

\section{MATERIALS AND METHODS}

This prospective study was approved by the Institutional Review Board of the Rabin Medical Center and adhered to the tenets of the Declaration of Helsinki. Informed consent was obtained from all participants before enrollment. Patients' companions arriving at the outpatient clinic of our center were recruited to the study. 
Only subjects with a healthy cornea were included. Patients with any ocular pathology, history of contact lens use, or previous surgeries were excluded from the study. All patients underwent slitlamp examination to rule out corneal pathologies, specifically the presence of guttae or pigment deposition on the endothelium, corneal dystrophies of any kind, and previous surgical interventions, such as laser iridotomy or refractive surgery. Once screened for our exclusion criteria through history and slitlamp examination, specular microscopy images were taken from healthy eligible subjects. Endothelial cell measurements were performed with both microscopes, after regular maintenance and factors calibration according to manufactures specifications. Each eye was captured three times by the same operator (D.J.), first with the KonanNoncon Robo SP-6000 and subsequently with the Tomey EM3000. The images were obtained in dim light environment while patients were asked to look at a central fixation target. Images with poor quality were excluded. Only the right eye of each patient was included to avoid biases arising from between-eye correlations.

The Konan-Noncon Robo SP-6000 SM system captures a field of $0.1 \mathrm{~mm}^{2}$ of the corneal endothelium at a magnification of $\times 180$, after autoalignment and automated image acquisition. Similar to previous reports, we used the center technique for manual counting of central endothelial images. ${ }^{8,9,11}$ The center of each cell, in a contiguous group of at least 100 cells, was manually marked and further analyzed by the device.

The Tomey EM-3000 SM uses an optical magnification of $\times 190$ and automatically captures 15 images of $0.135 \mathrm{~mm}^{2}$ field per measurement. ${ }^{8-10}$ The operator chose three high-quality central endothelial images for further analysis. Cell counting was performed using the manual (automated adjusted) center L-count method recommended by the Tomey EM-3000 manufacturer and used previously by other authors. ${ }^{6}$ Briefly, the device algorithm automatically marks the center of all cells in a variable-sized frame determined by the operator; then, the size of the frame changes to include at least 100 cells after which the operator can add or remove center markings. All cells lying within the discernible frame were manually marked with a central dot. Cells intersected by frame borders were marked and counted along two adjacent sides of the frame; cells along the two opposite sides of the frame were not counted (see Supplemental Fig, Supplemental Digital Content 1, http://links.lww.com/ICL/A138). In addition, the automated analysis was performed to allow further comparison with the Konan-Noncon Robo SP-6000 of other parameters in addition to ECD, such as hexagonality (\% HEX), average cell size (ACS), and coefficient of variation $(\mathrm{CoV})$. These are provided by the Tomey EM-3000 machine only in the automated mode. Data collected included the following: patient demographics, ECD, $\% \mathrm{HEX}, \mathrm{CoV}$, and ACS.

\section{Sample Size and Statistical Analysis}

Agreement of ECD readings between the two devices was evaluated through the Bland-Altman method. ${ }^{12}$ The limits of agreement (LoA) were defined as the mean interdevice difference \pm 1.96 SD. A folded empirical distribution function curve ("mountain plot") was used to describe the accumulative percentiles of measurements taken using the Tomey EM-3000 against their difference from their Konan-Noncon Robo SP-6000 paired reference value. ${ }^{13} \mathrm{~A}$ frequency analysis of the mean ECD reading differences was made using a frequency histogram. Intraclass correlation coefficient (ICC) served to assess intraobserver vari- ability (repeatability) for each device using a one-way random effects averaged measures model. The degree of reliability was classified according to the ICC as follows: slight $(0-0.2)$, fair (0.21-0.4), moderate (0.41-0.6), substantial (0.61-0.8), and almost perfect $(0.81-1) .{ }^{14}$ Statistical analysis was performed using SPSS, version 25.0. $P$ values $<0.05$ were considered statistically significant.

Power calculations showed that a minimum of 31 participants in a paired model were required to detect a clinically significant difference of 100 cells $/ \mathrm{mm}^{2}$, with a two-sided significance of 0.05 and power of 80 percent. SD was set at 200 cells $/ \mathrm{mm}^{2}$, based on previous studies comparing ECD counts among different devices. ${ }^{6}$

\section{RESULTS}

Forty-nine right eyes of 49 healthy subjects were included in the study. The mean patient age was $48.9 \pm 15.6$ (range: $21-80$ years) years, with 49 percent women. An average of $107.9 \pm 3.5$ cells (range: $100-123$ cells) and $104.3 \pm 7.6$ cells (range: $100-147$ cells) per image were included in the analysis for the Tomey EM-3000 and the Konan-Noncon Robo SP-6000 microscopes, respectively.

\section{Endothelial Cell Density}

The mean ECD was similar between the Tomey EM-3000 and the Konan-Noncon Robo SP-6000 SMs (2,713.2 \pm 242.4 vs. $2,700.8 \pm 300.5$ cells $\left./ \mathrm{mm}^{2}, P=0.47\right)$ with a mean difference of $12.4 \pm 119.6$ cells $/ \mathrm{mm}^{2}$ (95\% CI: -21.9 to +46.8 cells $\left./ \mathrm{mm}^{2}\right)$ and a mean absolute difference of $93.3 \pm 74.8$ cells $/ \mathrm{mm}^{2}$ (95\% CI: 71.8 to +114.7 cells $/ \mathrm{mm}^{2}$ ). The Bland-Altman plot showed that the 95\% LoA between the two machines ranged from -222.0 to 246.9 cells $/ \mathrm{mm}^{2}$ (Fig. 1). The ECD values derived by the two devices showed high correlation $(\mathrm{r}=0.92, P<0.0001)$.

Frequency analysis of ECD between the two SMs showed that $36.7 \%$ of eyes had a difference within \pm 50 cells $/ \mathrm{mm}^{2}$, and $63.3 \%$ of eyes had a difference within \pm 100 cells $/ \mathrm{mm}^{2}$ (Fig. 2). A folded empirical distribution function curve showed that all differences fell within 525.4 cells $/ \mathrm{mm}^{2}$, centered around a median of

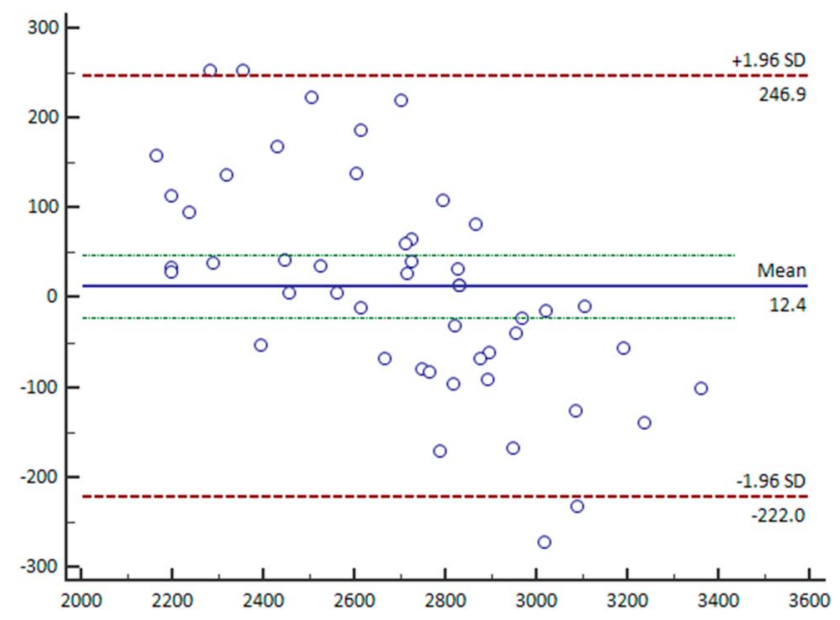

FIG. 1. Difference in ECD measurements of healthy subjects between the Tomey EM-3000 and the Konan-Noncon Robo SP-6000. The mean difference is represented by a solid line, $95 \%$ confidence interval by a green dotted line, and $95 \%$ limits of agreement by red dotted lines. 
13.3 cells $/ \mathrm{mm}^{2}$ (Fig. 3). Intraclass correlation coefficient calculation showed high repeatability for both the Konan-Noncon Robo SP-6000 (ICC 0.93; almost perfect) and for the Tomey EM-3000 microscope (ICC 0.88; almost perfect). Hexagonality, CoV, and ACS data are summarized in Table 1.

\section{DISCUSSION}

An accurate evaluation of the corneal endothelial layer is a critical step for appropriate clinical assessment and patient management. ${ }^{9,12,15,16}$ Although the Konan-Noncon Robo SP6000 and the Tomey EM-3000 SMs are widely used in clinical practice, there is a paucity of data regarding the interchangeability of the two devices. Our data show that the Konan-Noncon Robo SP-6000 and the Tomey EM-3000 are highly comparable when using the manual center technique and the manual L-count method analysis, respectively, with a mean ECD difference of only 12.4 cells $/ \mathrm{mm}^{2}$, a mean ECD absolute difference of 93.3 cells/ $\mathrm{mm}^{2}$, and low 95\% LoA. Repeatability analysis showed that both the Konan-Noncon Robo SP-6000 and the Tomey EM-3000 demonstrated a low intraobserver variability.

We opted to include in our study only healthy corneas to eliminate measurement and image interpretation biases that can result from inclusion of abnormal or postsurgery corneas. This allowed us to perform a net comparison between the two devices in more ideal conditions. In addition, to avoid biases arising from between-eye correlation, only the right eye of each patient was included. Our results were in agreement with previous studies showing good concordance between the two devices. Price et al. ${ }^{10}$ compared the endothelial readings of two noncontact SMs (Konan SP-8800 and the Tomey EM-3000) and one confocal microscope (Nidek ConfoScan 4), using five associated analysis methods for endothelial assessment in a mixed cohort of post-Descemet stripping endothelial keratoplasties and normal eyes. They found that, using cell identification methods, the Tomey EM-3000 automated software produced comparable ECD measurements with those obtained with the Konan SP-8800 and Nidek ConfoScan 4. Nevertheless, they could not report manual count using the Tomey EM3000 because the machine used in their study did not have an option for manual cell detection. In addition, only one goodquality image per patient was included in their analysis, compared with three images in our study. Luft et al. ${ }^{8}$ compared corneal endothelial parameters taken by four noncontact SMs, including the Konan CellChek XL and the Tomey EM-3000, in a mixed cohort of healthy, diseased, and postsurgery corneas. They also found a small ECD difference of 26 cells $/ \mathrm{mm}^{2}$ between the Tomey EM-3000 automatic count and the gold standard manual count of the Konan machine. However, in similar to the study by Price et al., the comparison between the Tomey EM-3000 and the Konan CellChek XL was made through different cell counting techniques, such as automated in the Tomey EM-3000 (manual counting feature was not available) versus manual in the Konan CellChek. ${ }^{10}$ Several dissimilarities exist between the study by Luft et al. and this study. Although Luft et al. aimed for three image acquisition by each machine investigated, this was not feasible in all cases. Therefore, in some of the cases, median values were considered for analysis when three images were taken, whereas in others, when two images were acquired, mean values were included. When only one image was captured, only the parameters of that image were included in the final analysis. In addition, both eyes of the same patient were included in their analysis. This by itself may cause statistical bias. By contrast, in our study, three good-quality images were analyzed along with inclusion of one eye for each patient and rapportage of mean values of three image readings for all subjects were included. Finally and most importantly, comparison between the two devices was made through manual counting technique. The manual technique is considered more accurate than the automatic mode ${ }^{10}$ because the automatic algorithm might skip some of the cells in a prefixed frame or alternatively mark one cell as more than a single cell. This misinterpretation is less likely to occur when a skilled and experienced operator manually analyses endothelial images. Our study can be regarded as complementary to outcomes from previous studies because comparison was performed through manual-to-manual analysis and not manual to automatic analysis.

Hexagonality, ACS, and $\mathrm{CoV}$ were also evaluated in our study. Only ACS was found to be comparable between the two machines. In similar to Luft et al., CoV data in our study show statistically significant difference between the Konan-Noncon Robo SP-6000 and the Tomey EM-3000 machines. However, in contrast to Luft et al. data, the difference in \%Hex between the two machines in our study was found to be significant. Hexagonality measurement is influenced by the health of the endothelium and by the mode of analysis of the image captured. This is demonstrated nicely by Luft et al., where a statistically significant difference was found in \%Hex between the manual and automated analysis of the Konan machine. Hexagonality data were provided only in the automatic mode of the Tomey EM-3000, and this was compared in our study with the \%Hex provided by the KonanNoncon Robo SP-6000 through the manual center technique. Noteworthy, Luft et al. did not find a statistically significant difference in the $\%$ Hex between the manual center technique of the Konan machine and the automatic mode of the Tomey EM-3000. This merits further investigation with a larger cohort and varying analysis methods.

When attempting to interpret corneal measurement using specular microscopy images, two important parameters typically

TABLE 1. Mean Hexagonal Cell Ratio (\%HEX), CoV, and ACS Correlation and Comparison of Values Provided by the Konan SP-6000 and the Tomey EM-3000

\begin{tabular}{|c|c|c|c|c|c|c|c|}
\hline & \multicolumn{3}{|c|}{ Comparison (Mean) } & \multicolumn{2}{|c|}{ Correlation } & \multicolumn{2}{|c|}{ Repeatability } \\
\hline & Konan SP-6000 & Tomey EM-3000 & $P$ & r & $P$ & Konan SP-6000 & Tomey EM-3000 \\
\hline HEX (\%) & $60.3 \pm 5.9$ & $46.2 \pm 8.7$ & $<0.0001$ & 0.29 & 0.04 & $\begin{array}{l}\text { ICC } \\
0.40 \text { (fair) }\end{array}$ & $\begin{array}{l}\text { ICC } \\
0.29 \text { (fair) }\end{array}$ \\
\hline $\begin{array}{l}\operatorname{ACS}(\mu \mathrm{m}) \\
\operatorname{CoV}\end{array}$ & $\begin{array}{l}375.2 \pm 43.0 \\
31.9\end{array}$ & $\begin{array}{l}371.9 \pm 34.5 \\
37.6\end{array}$ & $\begin{array}{l}0.19 \\
<0.001\end{array}$ & $\begin{array}{l}0.93 \\
0.31\end{array}$ & $\begin{array}{l}<0.001 \\
0.03\end{array}$ & ICC 0.93 (almost perfect) & ICC 0.89 (almost perfect) \\
\hline
\end{tabular}

\%Hex, hexagonal cell ratio; ACS, average cell size; CoV, coefficient of variation; ICC, intraclass correlation coefficient. 


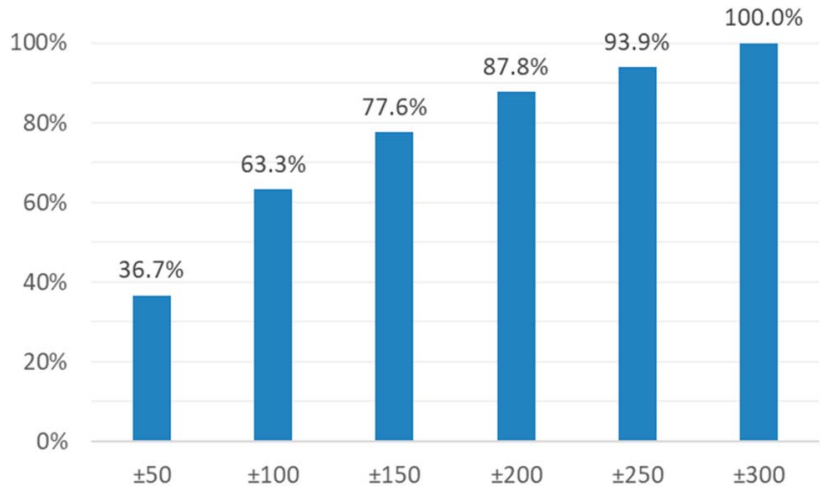

FIG. 2. Frequency histogram of ECD between the Tomey EM-3000 and the Konan-Noncon Robo SP-6000 specular microscopes. Histogram shows percentage of eyes in various difference intervals of ECD measurement.

influence accuracy: the device and the analytical method used. Currently, there is a consensus in the literature regarding the use of the semiautomated method over the automated method of the Konan cell check algorithm. This is widely reflected in all studies of the Cornea Donor Study Group, ${ }^{17}$ where high standards of eye banks and endothelial image reading centers for research and clinical purposes were implemented. Our mean absolute difference between the two devices $\left(93.3 \mathrm{cells} / \mathrm{mm}^{2}, 3.45 \%\right)$ is significantly lower than the $5 \%$ cutoff defined by the Cornea Donor Study Group as an interobserver variance standard for good-quality analysis while using the same noncontact SM, the Konan machine. Huang et al. ${ }^{16,18}$ compared the fully automatic mode with the semiautomatic mode of the Konan SP-9900 device using the center and flex-center methods in normal and glaucomatous eyes. They found that automated analysis tended to overestimate ECD in both healthy control and diseased eyes, compared with semiautomated (manual) analysis. ${ }^{16}$ Overestimating ECD may adversely affect treatment decisions and could lead to intraocular and postocular surgery-related complications. ${ }^{9}$ We have therefore used in our study only manual count for both devices. Noteworthy, in the L-count method used in our study for analysis of the Tomey EM-

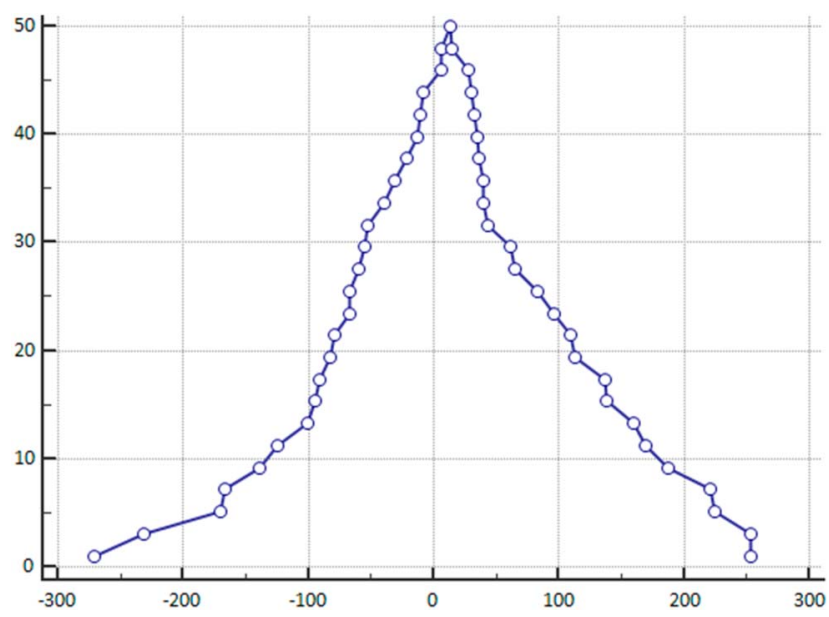

FIG. 3. Folded empirical distribution function plot depicting the Tomey EM-3000 measurements and the Konan SP-6000 measurements as a reference. The plot shows that all differences fall within 525.4 cells $/ \mathrm{mm}^{2}$ centered around a median of 13.3 cells $/ \mathrm{mm}^{2}$.
3000 images, cells are counted only along two adjacent sides of the frame. Another accepted analysis method is the fixed frame method, where all cells inside the frame are taken into consideration for final analysis, including cells intersecting all four sides of the frame. This might have resulted in higher cell counts of the Tomey EM-3000 machine and therefore result in less agreement between the devices.

Longitudinal assessment of patients requires measurements with high reproducibility, which is important for minimizing any variations arising from using different instruments or using the same device at different times. Luft et al. ${ }^{8}$ investigated four different SMs. Repeatability of ECD was evaluated through one-way analysis of variance testing by comparison of SD values. No statistically significant differences were observed between the devices investigated, with the Konan and the Tomey SMs having the highest SD values. This contrasts with Salvetat et al. ${ }^{19}$ who showed low intraobserver and interobserver test-retest variability in a sample of 42 healthy eyes using the Tomey EM-3000 automated analysis. Price et al. did not evaluate repeatability data. In our study, all three consecutive examinations and cell dotting with both instruments were performed by the same examiner. ${ }^{10} \mathrm{We}$ could therefore assess the intraobserver variability through ICC evaluation. The ICC for the Konan-Noncon Robo SP-6000 and the Tomey EM3000 was found to be high. Both the Konan-Noncon Robo SP6000 ICC and the Tomey EM-3000 ICC fall in the range of "almost perfect" repeatability. It is worth mentioning that the Konan had slightly higher ICC values when measuring repeatability and therefore is more reliable for longitudinal monitoring.

This study has several limitations. Patients with corneal guttae or post-keratoplasties were not included, and therefore, the results pertain only to healthy individuals. This limits the range of endothelial cell polymegathism and cell size variability. However, by selecting healthy individuals we eliminated possible biases in the acquisition and in the interpretation of endothelial cell images that might result from corneal morbidity. Further research is needed to evaluate the level of agreement between the two devices in nonhealthy corneas. The study strengths include its prospective design, the use of three images per subject for analysis, the fact that the same operator acquired all images negating any interobserver variability, the use of manual analysis method for both machines, and the use of a minimal count of 100 cells per image to increase accuracy.

In conclusion, our study shows that the difference in endothelial cell measurements between the Konan-Noncon Robo SP-6000 and the Tomey EM-3000 SMs through the center and the L-count analyzing techniques, respectively, is clinically small and not statistically significant. Nevertheless, caution should be taken when these machines are used interchangeably because the ECD difference between them can reach as high as 525.4 cells $/ \mathrm{mm}^{2}$.

\section{REFERENCES}

1. Doughty MJ. Could the coefficient of variation (COV) of the corneal endothelium be overestimated when a centre-dot method is used? Clin Exp Optom 2008;91:103-110.

2. Williams KK, Noe RL, Grossniklaus HE, et al. Correlation of histologic corneal endothelial cell counts with specular microscopic cell density. Arch Ophthalmol 1992;110:1146-1149.

3. Ohno K, Nelson LR, McLaren JW, et al. Comparison of recording systems and analysis methods in specular microscopy. Cornea 1999;18: 416-423. 
4. Benetz BA, Lass JH. Specular microscopy. Cornea 2018;37:S7-S8.

5. Corneal endothelial photography. Three-year revision. American Academy of Ophthalmology. Ophthalmology 1997;104:1360-1365.

6. Goldich Y, Marcovich AL, Barkana Y, et al. Comparison of corneal endothelial cell density estimated with 2 noncontact specular microscopes. Eur J Ophthalmol 2010;20:825-830.

7. de Sanctis U, Mutani B, Grignolo FM. Long-term endothelial cell loss after traumatic dislocation and repositioning of Artisan phakic IOL. J Refract Surg 2008;24:546-548.

8. Luft N, Hirnschall N, Schuschitz S, et al. Comparison of 4 specular microscopes in healthy eyes and eyes with cornea guttata or corneal grafts. Cornea 2015;34:381-386.

9. McCarey BE, Edelhauser HF, Lynn MJ. Review of corneal endothelial specular microscopy for FDA clinical trials of refractive procedures, surgical devices, and new intraocular drugs and solutions. Cornea 2008;27:1-16.

10. Price MO, Fairchild KM, Price FW Jr. Comparison of manual and automated endothelial cell density analysis in normal eyes and DSEK eyes. Cornea 2013;32:567-573.

11. Olsen T. Non-contact specular microscopy of human corneal endothelium. Acta Ophthalmol (Copenh) 1979;57:986-998.

12. Bland JM, Altman DG. Agreed statistics: Measurement method comparison Anesthesiology 2012;116:182-185.
13. Kitzmann AS, Winter EJ, Nau CB, et al. Comparison of corneal endothelial cell images from a noncontact specular microscope and a scanning confocal microscope. Cornea 2005;24:980-984.

14. Monti KL. Folded empirical distribution function curves-mountain plots. Am Statistician 1995;49:342-345.

15. Hong E, Ryu S, Kang M, et al. Comparison of repeatability of swept-source and spectral-domain optical coherence tomography for measuring inner retinal thickness in retinal disease. PLoS One 2019;16:e0210729.

16. Huang J, Maram J, Tepelus TC, et al. Comparison of manual \& automated analysis methods for corneal endothelial cell density measurements by specular microscopy. J Optom 2018;11:182-191.

17. Lass JH, Gal RL, Ruedy KJ, et al. An evaluation of image quality and accuracy of eye bank measurement of donor cornea endothelial cell density in the Specular Microscopy Ancillary Study. Ophthalmology 2005; 112:431-440.

18. Huang J, Liu X, Tepelus TC, et al. Comparison of the center and flex-center methods of corneal endothelial cell analysis in the presence of guttae. Cornea 2017;36:1514-1520.

19. Salvetat ML, Zeppieri M, Miani F, et al. Comparison between laser scanning in vivo confocal microscopy and noncontact specular microscopy in assessing corneal endothelial cell density and central corneal thickness. Cornea 2011;30:754-759. 